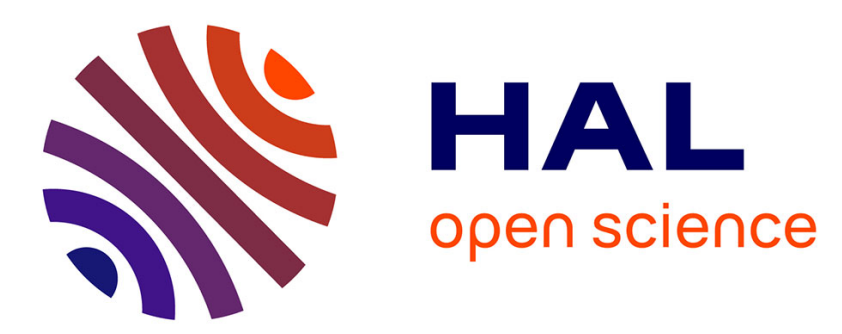

\title{
Degree of polarization estimation in the presence of nonuniform illumination and additive Gaussian noise
}

François Goudail, Mehdi Alouini, Daniel Dolfi

\section{To cite this version:}

François Goudail, Mehdi Alouini, Daniel Dolfi. Degree of polarization estimation in the presence of nonuniform illumination and additive Gaussian noise. Journal of the Optical Society of America. A Optics, Image Science, and Vision, 2008, 25 (4), pp.919-929. hal-00746712

\section{HAL Id: hal-00746712 \\ https://hal-iogs.archives-ouvertes.fr/hal-00746712}

Submitted on 29 Oct 2012

HAL is a multi-disciplinary open access archive for the deposit and dissemination of scientific research documents, whether they are published or not. The documents may come from teaching and research institutions in France or abroad, or from public or private research centers.
L'archive ouverte pluridisciplinaire HAL, est destinée au dépôt et à la diffusion de documents scientifiques de niveau recherche, publiés ou non, émanant des établissements d'enseignement et de recherche français ou étrangers, des laboratoires publics ou privés. 


\title{
Degree of polarization estimation in the presence of nonuniform illumination and additive Gaussian noise
}

\author{
Arnaud Bénière, ${ }^{1,2}$ François Goudail, ${ }^{1, *}$ Mehdi Alouini, ${ }^{2}$ and Daniel Dolfi ${ }^{2}$ \\ ${ }^{1}$ Laboratoire Charles Fabry de l'Institut d'Optique, CNRS, Univ Paris-Sud, Campus Polytechnique, RD 128, \\ 91127 Palaiseau, France \\ ${ }^{2}$ Thales Research and Technology_France, RD128, 91767 Palaiseau Cedex, France \\ *Corresponding author: francois.goudail@institutoptique.fr
}

Received August 24, 2007; revised January 25, 2008; accepted January 31, 2008; posted February 11, 2008 (Doc. ID 86790); published March 24, 2008

\begin{abstract}
Within the general framework of active imaging we address the degree of polarization (DOP) estimation in the presence of additive Gaussian detector noise. We first study the performance of standard DOP estimators and propose a method to increase estimation precision using physically relevant a priori information. We then consider the realistic case of nonuniform illumination distribution. We derive the Cramer-Rao lower bound and determine a profile likelihood-based estimator. We demonstrate the efficiency of this new estimator and compare its performance with other standard estimators as a function of the degree of nonuniformity of the illumination. (C) 2008 Optical Society of America
\end{abstract} OCIS codes: 260.543, 030.4280 .

\section{INTRODUCTION}

Imaging systems that measure the degree of polarization (DOP) have been a topic of growing interest in several domains such as machine vision [1], biomedical imaging [2], and remote sensing [3]. These systems can for example reveal contrasts between parts of a scene that have the same intensity reflectivity but different polarimetric properties [4].

One of the simplest active polarimetric imaging principles consists of illuminating the scene with a totally polarized light beam and computing the orthogonal state contrast image (OSCI) from two intensity images of the same scene. The first image is formed with the fraction of the backscattered light polarized parallel to the incident light, and a second one with light orthogonal to the incident one. It should be noted that the polarization state of the incident light need not be linear, but may be any pure polarization state on the Poincaré sphere. Moreover, it has been shown in [5] that the OSCI is an estimate of the DOP of the backscattered light if the observed materials are purely depolarizing. We will make this assumption in the remainder of this paper.

However, the intensity measurements used to compute the DOP images are corrupted with noise, and it is necessary to take this into account in order to improve the performance of DOP estimators. The estimation of phase delay has been studied in the presence of additive Gaussian noise coupled to photon counting noise [6]. Concerning DOP estimation, the influence of speckle noise [7] and of coupled speckle and photon noise in low-flux-intensity images [8] has been studied. More recently, we have addressed the case of additive Gaussian noise [9]. We have shown in particular that this type of noise poses specific problems at low SNR since the variances of usual estimators diverge.

In this paper, we consider that illumination is weakly coherent, so the speckle noise is not dominant and may be neglected. This assumption has been verified for the wideband light sources we will use, but also with the highpower laser sources used in [10], which do not lead to speckled images. We thus focus on two important issues relative to the estimation of DOP when the intensity images used to form the DOP image are perturbed by detector noise, which can be modeled as additive and Gaussian [9]. First, we propose and characterize a way of avoiding the divergence of DOP estimators when the SNR is low in the intensity images. Second, we address the problem of nonuniform illumination of the scene. Indeed, in DOP imaging, the illumination of the scene is often nonuniform owing to a spatially or temporally nonuniform light source. Standard estimators of the DOP assume that the illumination is the same on all pixels of the sample. We propose a method to reliably estimate the DOP when this hypothesis is not fulfilled.

The paper is organized as follows. In Section 2, we review the main results on DOP estimation precision in the presence of additive Gaussian noise and uniform illumination. In Section 3, we compare the performance of several DOP estimators in terms of bias and standard deviation and show that it is possible to efficiently regularize the maximum-likelihood (ML) estimator by using physically relevant a priori information. Section 4 is devoted to the nonuniform illumination case. We determine the Cramer-Rao lower bound (CRLB) and the profilelikelihood estimator. We demonstrate the superiority of this new estimator over standard ones and characterize 
its performance as a function of the degree of nonuniformity of the illumination.

\section{DOP ESTIMATION IN THE PRESENCE OF ADDITIVE GAUSSIAN NOISE}

A simple but efficient active polarimetric active imaging mode can consist of illuminating the scene with a totally polarized beam and forming two spatial or temporal data samples relative to the same object in the scene [11-13]. The first one $\mathbf{X}=\left\{X_{i}, i \in[1, N]\right\}$ is formed with the backscattered light in the same state of polarization as the incident light. The second one $\mathbf{Y}=\left\{Y_{i}, i \in[1, N]\right\}$ is formed with the light polarized orthogonally to the incident state. One can gather those two sets of measurements in a the statistical sample $\chi=\{\mathbf{X}, \mathbf{Y}\}$. This sample can be spatial if we consider a neighborhood of pixels corresponding to the same object in the scene, or temporal if we consider a single pixel in several acquisitions. We assume that $\mathbf{X}$ and $\mathbf{Y}$ are polluted with an additive Gaussian noise and that the incident beam is noncoherent temporally and spatially uniform. One thus has two vectors of random variables:

$$
\begin{aligned}
& X_{i}=m_{X}+n_{i}^{x}, \\
& Y_{i}=m_{Y}+n_{i}^{y},
\end{aligned}
$$

where $m_{X}$ and $m_{Y}$ are the true values and each measure is perturbed by additive noises $n_{i}^{x}$ and $n_{i}^{y}$ whose probability-density functions (PDF) are assumed Gaussian with zero mean and variance $\sigma^{2}$. The noises $n_{i}^{x}$ and $n_{i}^{y}$ are assumed statistically independent. We will assume that the materials present in the scene only depolarize light without otherwise affecting the state of polarization. In this case, the degree of polarization of the light backscattered by the scene is defined as

$$
P=\frac{m_{X}-m_{Y}}{m_{X}+m_{Y}} .
$$

The parameters of the problem are the two average values $m_{X}$ and $m_{Y}$. If one considers the total intensity $I$ $=m_{X}+m_{Y}$, one can define another parametrization $(I, P)$ where $m_{X}=I(1+P) / 2$ and $m_{Y}=I(1-P) / 2$.

A first approach to determine the potential precision estimation of the DOP is to compute the CRLB. The CRLB is a lower bound on the variance that may be obtained by an unbiased estimator. Taking into account the noise model in Eq. (2) the expression of the CRLB of parameter $P$ is [9]

$$
C R L B=\frac{\left(1+P^{2}\right)}{S N R^{2}},
$$

where

$$
S N R=\frac{I \sqrt{N}}{\sqrt{2} \sigma}
$$

corresponds to the averaged SNR on the intensity image. The CRLB is independent of the estimator and thus characterizes the estimation problem. It can thus serve as a benchmark to evaluate the performance of actual unbiased estimators.

A classical way to determine good estimators is the Maximum-Likelihood (ML) principle. The ML estimator is not always efficient for a fixed number $N$ of data, but it has good asymptotic properties as $N$ increases [14]. The ML estimator of $P$ can be shown to have the expression [9]

$$
\hat{P}_{m l}=\frac{\sum_{i=1}^{N} X_{i}-\sum_{i=1}^{N} Y_{i}}{\sum_{i=1}^{N} X_{i}+\sum_{i=1}^{N} Y_{i}} .
$$

By using the above defined characteristics of the random variables $X_{i}$ and $Y_{i}$, one can express this estimator as

$$
\hat{P}_{m l}=\frac{S N R \times P+b_{1}^{\prime}}{S N R+b_{2}^{\prime}},
$$

where $S N R$ is defined in Eq. (4) and $P$ the true value of the DOP. The variables $b_{1}^{\prime}$ and $b_{2}^{\prime}$ are two independent Gaussian random variables with zero mean and a variance of 1 .

Equation (6) shows that $\hat{P}_{m l}$ is the ratio of two normal random variables. The probability-density function (PDF) of this type of random variables has been determined in [15]. By using the results therein, we have shown in [9] that the PDF of $\hat{P}_{m l}$ can be expressed as

$$
\mathcal{P}(\rho)=\alpha \mathcal{P}_{1}(\rho)+(1-\alpha) \mathcal{P}_{2}(\rho),
$$

where $\alpha=\exp \left[-S N R^{2}\left(1+P^{2}\right) / 2\right]$ and

$$
\begin{aligned}
\mathcal{P}_{1}(\rho)= & \frac{1}{\pi\left(1+\rho^{2}\right)}, \\
\mathcal{P}_{2}(\rho)= & \frac{S N R}{\sqrt{2 \pi}(1-\alpha)} \frac{1+\rho P}{\left(1+\rho^{2}\right)^{3 / 2}} \mathrm{~F}\left[S N R \frac{(1+\rho P)}{\sqrt{1+\rho^{2}}}\right] \\
& \times \exp \left[-\frac{S N R^{2}(\rho-P)^{2}}{2\left(1+\rho^{2}\right)}\right],
\end{aligned}
$$

with $\mathrm{F}(x)=[\operatorname{erf}(x / \sqrt{2})-\operatorname{erf}(-x / \sqrt{2})] / 2, \quad$ and $\operatorname{erf}(x)$ $=2 / \sqrt{\pi} \int_{0}^{x} \exp \left(-t^{2}\right) \mathrm{d} t$ is the classical error function.

This expression shows that the PDF of $\hat{P}_{m l}$ is the weighted sum of two PDF. The first one $\mathcal{P}_{1}$ is the Cauchy PDF [16]. It is known that all its statistical moments, including average and variance, are not defined. Its main characteristic is to lead to very large deviations with significant probability, which makes it a noise particularly difficult to handle. Thanks to the exponential factor, all statistical moments of the PDF $\mathcal{P}_{2}$ are defined. One can see on Fig. 1 that as $S N R$ increases, $\mathcal{P}$ gets close to a Gaussian PDF with a mean value equal to $P$ and variance equal to $\left(1+P^{2}\right) / S N R^{2}$ (that is, to the CRLB). For low SNR, the PDF is close to a Cauchy law which is not even centered on the true value of the DOP.

\section{IMPROVEMENT OF DOP ESTIMATION}

In this section, we will compare different estimators of the DOP and propose an efficient method to regularize the ML estimator based on physically relevant $a$ priori knowledge. 


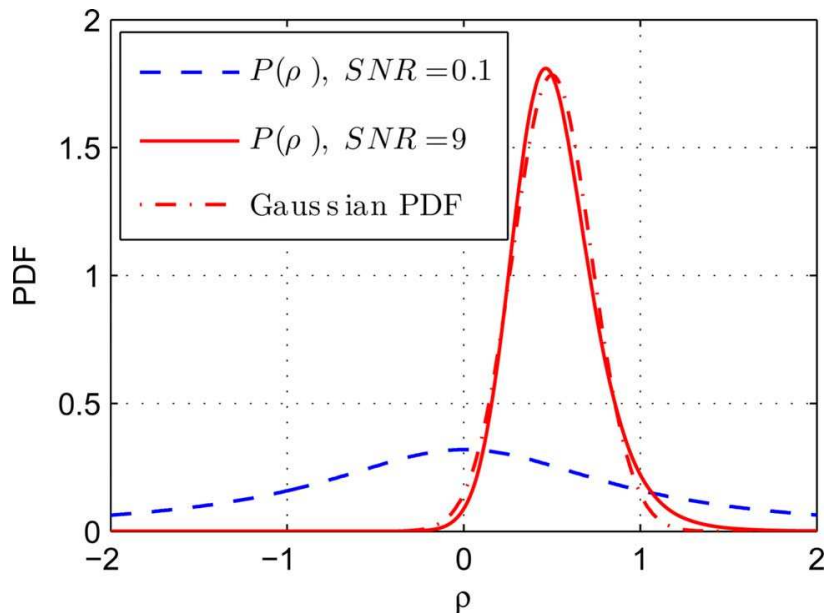

Fig. 1. (Color online) PDF $\mathcal{P}$ [see Eq. (7)] for two values of the $\mathrm{SNR}$ and $\mathrm{P}=0.5$; Gaussian $\mathrm{PDF}$ with variance $\left(1+P^{2}\right) /\left(S N R^{2}\right)$ for $\mathrm{SNR}=9, \mathrm{P}=0.5$.

\section{A. Comparison of DOP Estimators}

Besides the ML estimator defined in the previous section, let us first consider the two following estimators:

$$
\begin{aligned}
\hat{P}_{e m} & =\frac{1}{N} \sum_{i=1}^{N} \frac{X_{i}-Y_{i}}{X_{i}+Y_{i}}, \\
\hat{P}_{\text {med }} & =\operatorname{median}\left(\frac{X_{i}-Y_{i}}{X_{i}+Y_{i}}\right) .
\end{aligned}
$$

The estimator $\hat{P}_{e m}$ consists of computing the empirical mean of the pixelwise estimates of the DOP. The estimator $\hat{P}_{\text {med }}$ is the median of the sample constituted of the pixelwise estimates of the DOP. We estimate the empirical means and standard deviations of these estimators with Monte Carlo simulations and compare them with those of the ML estimator in Fig. 2. The value of $\mathrm{P}$ is set to 0.5 and the size of the sample to $\mathrm{N}=9$, which corresponds, for example, to temporally averaging nine acquisitions.

It is observed that empirical means and standard deviations of both the ML and the empirical mean estima- tors diverge at low SNR. This is because the probability of having a large, Cauchy-like deviation in some of the $10^{4}$ generated samples is not negligible. For higher SNR values, the biases of both estimators tend to zero, and their standard deviations tend to the CRLB. However, it is noted that the ML estimator becomes "well-behaved" for much lower values of the SNR than the empirical mean estimator, which makes the former preferable to use.

On the other hand, the estimated mean and standard deviation of the median estimator do not diverge for low SNR. Indeed, the median of a Cauchy law is defined and equal to zero, and this is why the estimated mean of $\hat{P}_{\text {med }}$ tends toward zero for low SNR. That its standard deviation goes lower than the CRLB for very low SNR is theoretically possible, as the estimator is biased. However, in every other case, it is noted that this estimator always yields a higher standard deviation than the CRLB, and thus than the ML, even at higher SNR.

\section{B. Experimental Results}

To illustrate these conclusions, we have performed the following experiments with a Basler A312f, 12 bit camera. A piece of homogeneous diffusive plastic is illuminated with a linearly polarized beam from an incoherent light source. The CCD delivers only positive gray levels, but we have added an offset during the acquisition, which is a common experimental practice especially when low intensity levels must be detected. When the offset is subtracted after the measure, one obtains a Gaussian distribution with negative values for low $S N R$. Moreover, at low $S N R$, it may happen that the two measured values $X_{i}$ and $Y_{i}$ are such that $X_{i}+Y_{i}=0$, so that the value of the estimated $P$ is either infinite or not defined. We choose not to take those measurements into consideration.

Two samples $\mathbf{X}$ and $\mathbf{Y}$ are acquired, corresponding respectively to the backscattered light in the same polarization state as the incident beam and light polarized orthogonally to the incident state. Those samples contain $50 \times 50$ pixels and are acquired successively 9 times. The system aperture is deliberately reduced to obtain low SNR values and mimic operational situations of longrange object detection. We thus have 2500 realizations of
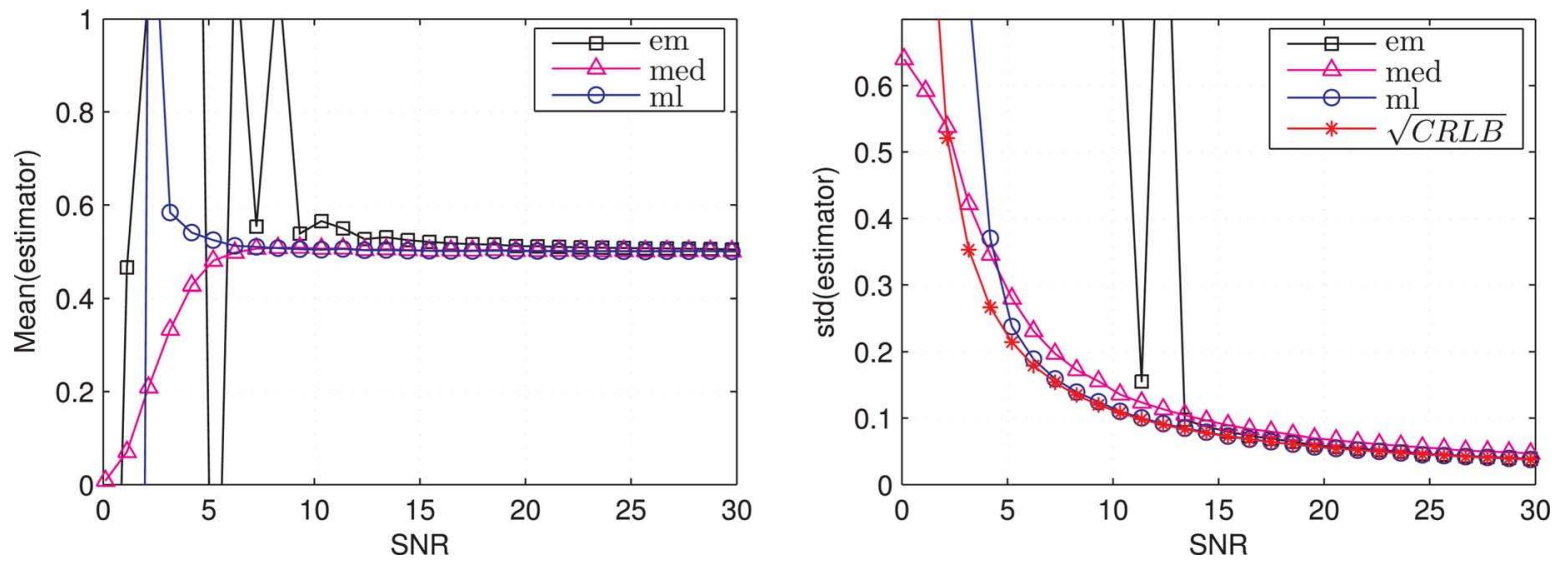

Fig. 2. (Color online) Estimated means; standard deviations of estimators $\hat{P}_{e m}, \hat{P}_{\text {med }}, \hat{P}_{m l}$; and square root of CRLB for $N=9$ samples and $\mathrm{P}=0.5$. The estimations are made with $10^{4}$ realizations. 
the random vectors $\mathbf{X}=\left\{X_{i}, i \in[1, N]\right\}$ and $\mathbf{Y}=\left\{Y_{i}, i\right.$ $\in[1, N]\}$ with $N=9$. To estimate the mean and standard deviation of the estimators we will use these 2500 realizations.

The histograms, the estimated mean, and the standard deviations of the DOP estimators $\hat{P}_{\text {med }}, \hat{P}_{m l}$, and $\hat{P}_{m l t}$ appear on Fig. 3. In Table 1 we compare these experimental results with Monte Carlo simulations and observe that they are in good agreement. One notices, however, that the results of the empirical mean estimator are better than those indicated by the Monte Carlo simulations: This is because we did not consider the measurements that lead to infinite or not defined estimates of the DOP, which artificially reduces the standard deviation of this estimator.

\section{Improvement of the ML Estimator}

On the histograms of Fig. 3, we can see that all the estimators considered can lead to estimated values of $P$ that are negative or larger than 1 . Such values are physically unrealistic. Indeed, it is seen from the definition of $P$ in Eq. (2) that it must be smaller than 1. Moreover, if the materials in the scene only depolarize light, one must have $m_{X}>m_{Y}$. Indeed, when noise is absent, there cannot be more light in the orthogonal state than in the parallel state: Equality happens when the light backscattered by the scene is totally depolarized.

It is possible to take into account this knowledge to improve the performance of the ML estimator. Let us define the new "truncated" estimator as

$$
\begin{aligned}
\hat{P}_{m l t}=\hat{P}_{m l} \quad \text { if } \hat{P}_{m l} \in[0,1] ; \\
=0 \quad \text { if } \hat{P}_{m l}<0 ; \\
=1 \quad \text { if } \hat{P}_{m l}>1 .
\end{aligned}
$$

In order to characterize its performance, we have performed Monte Carlo simulations and estimated the bias

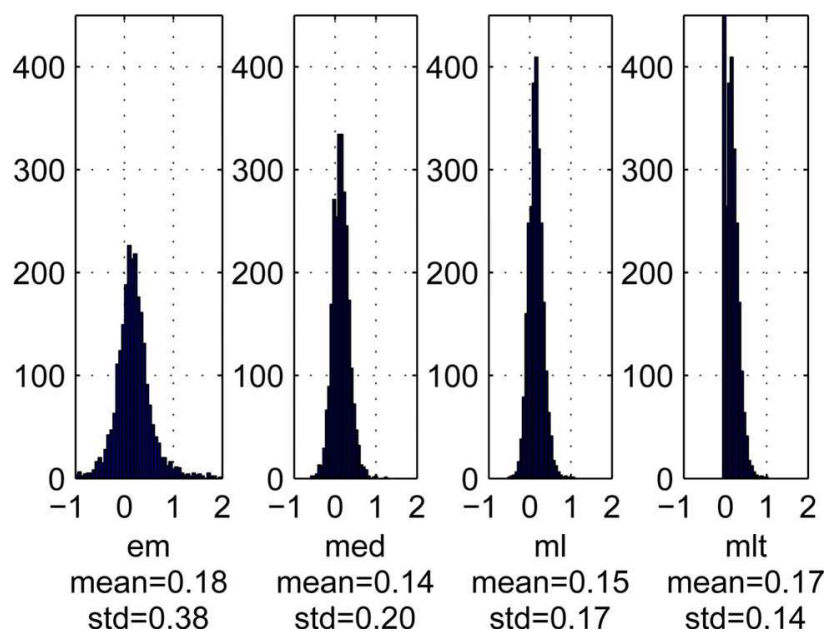

Fig. 3. (Color online) Histograms of $\hat{P}_{e m}, \hat{P}_{m e d}, \hat{P}_{m l}$, and $\hat{P}_{m l t}$ [see Eq. (8), Eq. (9), Eq. (5), and Eq. (10)], obtained with $\mathrm{N}=9$ and 2500 realizations. The conditions of acquisition with a Basler A312f camera were such that $S N R \simeq 6.1$ and the real value of the DOP was $\mathrm{P}=0.15$ (this value has been estimated at high $S N R$ ).
Table 1. Estimated Mean and Standard Deviation of the Empirical Mean $\left(\hat{\boldsymbol{P}}_{\text {em }}\right)$, the Median $\left(\hat{\boldsymbol{P}}_{\text {med }}\right)$, the Maximum Likelihood $\left(\hat{\boldsymbol{P}}_{m l}\right)$, and the Truncated Maximum Likelihood $\left(\hat{\boldsymbol{P}}_{\text {mlt }}\right)$ Estimators ${ }^{a}$

\begin{tabular}{lcccc}
\hline & \multicolumn{4}{c}{ Estimator } \\
\cline { 2 - 5 } Estimation & $\hat{P}_{e m}$ & $\hat{P}_{\text {med }}$ & $\hat{P}_{m l}$ & $\hat{P}_{m l t}$ \\
\hline Mean & & & & \\
Experimental & 0.18 & 0.14 & 0.15 & 0.17 \\
Simulated & 0.25 & 0.14 & 0.15 & 0.17 \\
Standard Deviation & & & & \\
Experimental & 0.38 & 0.20 & 0.17 & 0.14 \\
Simulated & 3.81 & 0.21 & 0.17 & 0.15 \\
\hline
\end{tabular}

${ }^{a}$ The experimental results are those obtained in Fig. 3. The simulated results are obtained by Monte Carlo simulations with $N=9,2500$ realizations, $\mathrm{SNR}=6.1$, and a true value of DOP $P=0.15$.

$b=P-\langle\hat{P}\rangle$, the standard deviation $\sigma=\sqrt{\left\langle(P-\hat{P})^{2}\right\rangle}$, and the root mean square deviation $\mathrm{RMSD}=\sqrt{b^{2}+\sigma^{2}}$. The RMSD is a way of representing the compound effect of bias and variance on the global deviation of an estimator.

The results are presented in Fig. 4. The estimated bias and standard deviation of the estimators for $P=0.1$ are in perfect agreement with the experiments illustrated on Fig. 3. It is seen that $\hat{P}_{m l t}$ naturally does not diverge anymore, and its RMSD is always lower than that of the ML estimator. It is even noted that the standard deviation of $\left\langle\hat{P}_{m l t}\right\rangle$ can be lower than the CRLB, which is theoretically possible as this estimator is biased. Moreover, according to the simulations, $\left\langle\hat{P}_{m l t}\right\rangle$ converges to a value about 0.36 as the $S N R$ decreases toward 0 . This value can be obtained theoretically, as described in Appendix A. It can thus be concluded that the $\hat{P}_{m l t}$ improves the estimation performance and efficiently avoids aberrant results. It should thus be used whenever the physical hypotheses from which it is derived are fulfilled.

\section{ESTIMATION OF THE DOP IN THE PRESENCE OF NONUNIFORM ILLUMINATION}

The ML estimator described in the previous section is based on the hypothesis that all the pixels in the sample have the same mean values $m_{X}$ and $m_{Y}$ [see Eq. (2)]. If the reflectivity of the material is assumed homogeneous inside the sample, this corresponds to also assuming that the illumination is uniform. However, this may not be the case in practice. Indeed, the beam that illuminates the scene may be spatially nonuniform, and the illumination can also be temporally nonuniform especially if a pulsed illumination system is considered.

In this section, we thus address the problem of estimation of the DOP in the presence of nonuniform illumination. We first define a new statistical model that takes into account the nonuniform illumination as a nuisance parameter. We then determine the CRLB and the ML estimator when the illumination is nonuniform but known, to serve as a benchmark. We finally determine the profile likelihood estimator adapted to cases where the illumina- 

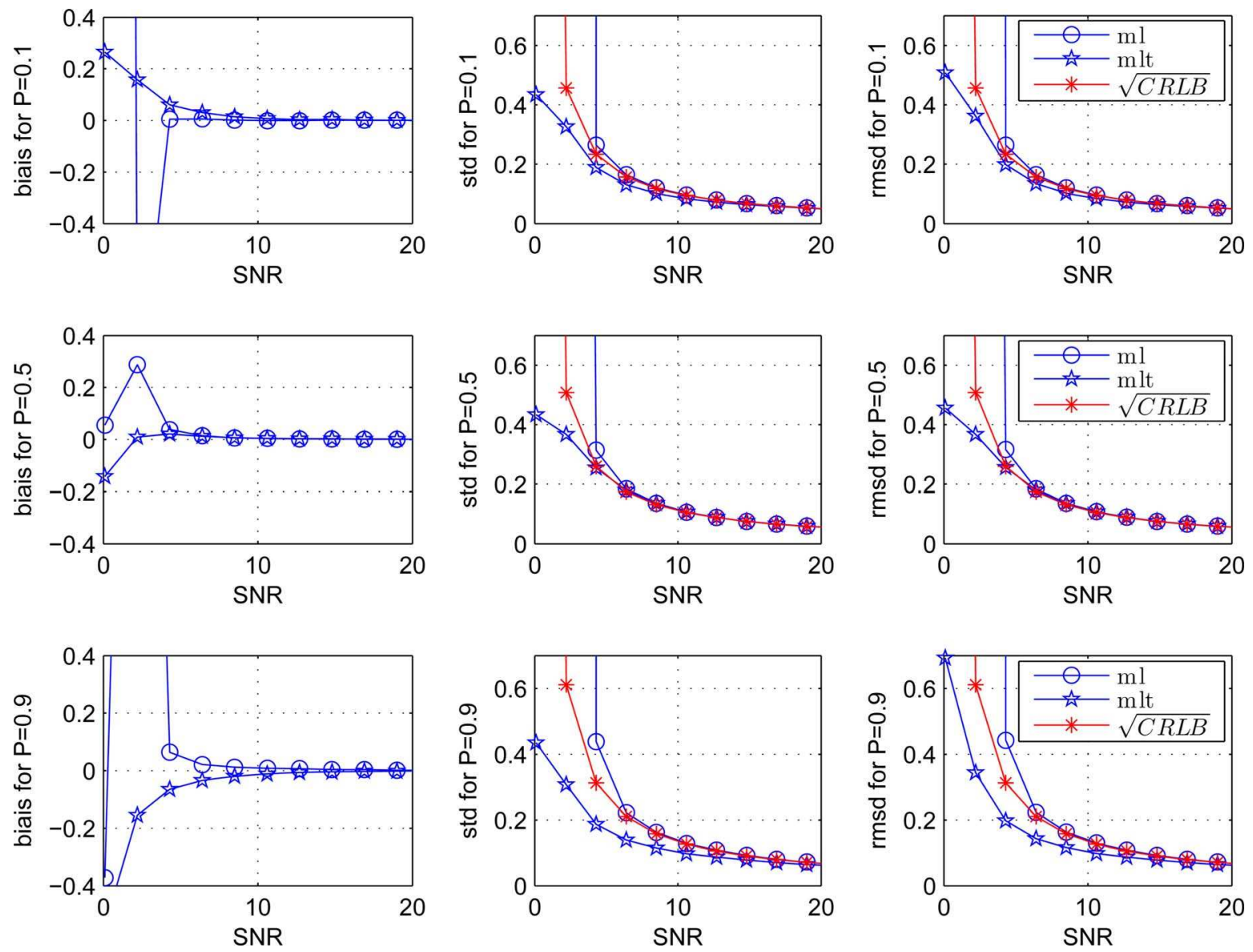

Fig. 4. (Color online) Estimated bias, standard deviation, and root mean square deviation of estimators $\hat{P}_{m l}$ and $\hat{P}_{m l t}$ with 25 samples as a function of the SNR and square root of the CRLB. We performed Monte Carlo simulations with $10^{4}$ realizations for three different values of the DOP: first row, $\mathrm{P}=0.1$; second row, $\mathrm{P}=0.5$; third row: $\mathrm{P}=0.9$.

tion is unknown. We analyze its performance as a function of the illumination nonuniformity and demonstrate that it is more efficient than standard DOP estimators.

\section{A. Image Model}

The measured data $N$-sample $\chi^{\prime}=\left(\mathbf{X}^{\prime}, \mathbf{Y}^{\prime}\right)$ is modeled as

$$
\begin{aligned}
& X_{i}=F_{i} m_{X}+n_{i}^{x}, \\
& Y_{i}=F_{i} m_{Y}+n_{i}^{y},
\end{aligned}
$$

for $i \in[1, N]$, where $m_{X}$ and $m_{Y}$ are the true values. Note that the observed sample is still considered homogeneous, that is, all pixels are described by the same values $m_{X}$ and $m_{Y}$. Only the illumination is assumed nonhomogeneous. The parameter to estimate is still $P=\left(m_{x}-m_{y}\right) /\left(m_{x}+m_{y}\right)$. Each measure is perturbed by the noises $n_{i}^{x}$ and $n_{i}^{y}$ whose PDF are assumed Gaussian with zero mean and variance $\sigma^{2}$. The noises $n_{i}^{x}$ and $n_{i}^{y}$ are assumed statistically independent. The vector $\mathbf{F}$ elements are positive and denote the spatial or temporal variations of the illumination intensity. We will first assume that this vector is known to the user. This hypothesis is relevant when the nonuniformity is spatial, and when a calibration of the system has been performed. In the case of temporal nonuniformity related to pulse-to-pulse fluctuations this hypothesis becomes unrealistic. However the results obtained above can be used as a benchmark. We will then address estimation in the more realistic case where $\mathbf{F}$ is unknown.

\section{B. CRLB with Nonuniform but Known Illumination}

Our objective is to determine the CRLB on P. For that purpose, we determine the elements of the Fisher Matrix I which is defined by

$$
\boldsymbol{I}=\left[\begin{array}{cc}
\left\langle-\frac{\partial^{2} l}{\partial I^{2}}\right\rangle & \left\langle-\frac{\partial^{2} l}{\partial I \partial P}\right\rangle \\
\left\langle-\frac{\partial^{2} l}{\partial P \partial I}\right\rangle & \left\langle-\frac{\partial^{2} l}{\partial P^{2}}\right\rangle
\end{array}\right],
$$

where $l$ is the log-likelihood and $\langle$.$\rangle denotes statistical av-$ eraging. Let us start with the expression of the loglikelihood determined from the model Eq. (11):

$$
\begin{aligned}
l\left(\chi^{\prime} \mid \boldsymbol{F}, m_{X}, m_{Y}\right)= & -2 N \ln (\sqrt{2 \pi} \sigma)-\frac{1}{2 \sigma^{2}} \sum_{i=1}^{N}\left(X_{i}-F_{i} m_{X}\right)^{2} \\
& -\frac{1}{2 \sigma^{2}} \sum_{i=1}^{N}\left(Y_{i}-F_{i} m_{Y}\right)^{2}
\end{aligned}
$$

Using the $(I, P)$ parametrization one has: 


$$
\begin{aligned}
l\left(\chi^{\prime} \mid \boldsymbol{F}, I, P\right)= & -2 N \ln (\sqrt{2 \pi} \sigma)-\frac{1}{2 \sigma^{2}} \sum_{i=1}^{N}\left(X_{i}-F_{i} \frac{I(1+P)}{2}\right)^{2} \\
& -\frac{1}{2 \sigma^{2}} \sum_{i=1}^{N}\left(Y_{i}-F_{i} \frac{I(1-P)}{2}\right)^{2} .
\end{aligned}
$$

Consequently, the matrix $\boldsymbol{J}$ that corresponds to the inverse of the Fisher matrix has the expression

$$
\mathbf{J}=\mathbf{I}^{-1}=\frac{2 \sigma^{2}}{\sum_{i=1}^{N} F_{i}^{2}}\left[\begin{array}{cc}
1 & -\frac{P}{I} \\
-\frac{P}{I} & \frac{\left(1+P^{2}\right)}{I^{2}}
\end{array}\right] .
$$

The CRLB on the estimation of $\mathrm{P}$ is given by the lower right element of $\mathbf{J}[14]$ :

$$
C R L B_{F}=\frac{2 \sigma^{2}}{\sum_{i=1}^{N} F_{i}^{2}} \frac{\left(1+P^{2}\right)}{I^{2}}=\frac{\left(1+P^{2}\right)}{S N R_{F}^{2}},
$$

with

$$
S N R_{F}=\frac{I \sqrt{\sum_{i=1}^{N} F_{i}^{2}}}{\sqrt{2} \sigma} .
$$

This expression is very similar to that of the CRLB in the case of uniform illumination defined in Eq. (3). The SNR has just be replaced by $S N R_{F}$, which takes into account the illumination vector $\mathbf{F}$. Indeed, we observe that if $F_{i}=1, \forall i \in[1, N]$, one has the same expression as in the uniform illumination case.

\section{Potential Gain in Precision When Taking into Account the Nonuniformity}

Considering a given level of illumination nonuniformity, our objective now is to evaluate the gain in precision due to taking into account this nonuniformity in the image model. For that purpose, we study the CRLB in both uniform and nonuniform illumination. This will give us an idea of the potential precision we can expect with both image models in the presence of nonuniform illumination.

Let us consider a given value of the total illumination intensity in the sample, that is, $\sum_{i=1}^{N}\left(m_{X} F_{i}+m_{Y} F_{i}\right)=I F^{0}$. Using the Cauchy-Schwartz inequality and the fact that $\forall i, F_{i}>0$, one has $\left(F^{0}\right)^{2} / N \leqslant \Sigma_{i=1}^{N} F_{i}^{2} \leqslant\left(F^{0}\right)^{2}$ and thus

$$
S N R_{F}^{\min } \leqslant S N R_{F} \leqslant S N R_{F}^{\max }
$$

with $S N R_{F}^{\min }=I F^{0} / \sqrt{2} \sigma / \sqrt{N}$ and $S N R_{F}^{\max }=I F^{0} / \sqrt{2} \sigma$. The lower bound $S N R_{F}^{\min }$ corresponds to the uniform illumination $F_{i}=F^{0} / N, \forall i$. The upper bound $S N R_{F}^{\max }$ corresponds to an illumination concentrated on a single sample $j$ : $F_{j}=F^{0}$ and $\forall i \neq j, F_{i}=0$. In practice, this means that if we have a certain amount of light $I F^{0}$ available for $N$ acquisitions, we will reach the highest $S N R_{F}$ by concentrating the light in one pulse for the first acquisition even if the $N-1$ other acquisitions do not have any illumination. It can be shown (see Appendix B) that if we cope with nonuniform illumination by adopting the uniform model presented in Section 2, a lower bound on estimation variance is

$$
C R L B_{U}=\frac{1+P^{2}}{\left(S N R_{F}^{\min }\right)^{2}} .
$$

Let us now compare $C R L B_{F}$ with $C R L B_{U}$, that is, find the potential gain in estimation precision if we use the nonuniform model instead of the uniform one. One has

$$
C R L B_{U}=C R L B_{F}\left(\frac{S N R_{F}}{S N R_{F}^{\min }}\right)^{2}=C R L B_{F}\left(1+\frac{\sigma_{F}^{2}}{m_{F}^{2}}\right),
$$

where $m_{F}=(1 / N) \sum_{i=1}^{N} F_{i}$ is the empirical mean of the illumination and $\sigma_{F}^{2}=(1 / N) \Sigma_{i=1}^{N} F_{i}^{2}-m_{F}{ }^{2}$ is its empirical variance. We know that $\sigma_{F}^{2} / m_{F}^{2}$ varies between 0 and $\mathrm{N}-1$. Let us define the quantity $Q$ that characterizes the nonuniformity of the illumination and varies between 0 and 1:

$$
Q=\frac{1}{N-1} \frac{\sigma_{F}^{2}}{m_{F}^{2}} \text {. }
$$

We thus have

$$
C R L B_{U}=C R L B_{F}[1+(N-1) Q] .
$$

The square roots of these CRLBs are plotted on Fig. 5 in dashed and dotted-dashed curves. They represent the potential precision that can be reached by an unbiased estimator based on, respectively, a uniform and a nonuniform illumination model.

\section{Examples}

To illustrate the gain in precision when using the nonuniform model, let us consider some typical distributions of illumination. Let us compare $s t d_{U}=\sqrt{C R L B_{U}}$, the potential precision using the uniform model, and $s t d_{F}$ $=\sqrt{C R L B_{F}}$, the potential precision using a nonuniform model. Consider:

(1) Deterministic uniform illumination: $F_{i}=F_{j}, \forall i, j$.

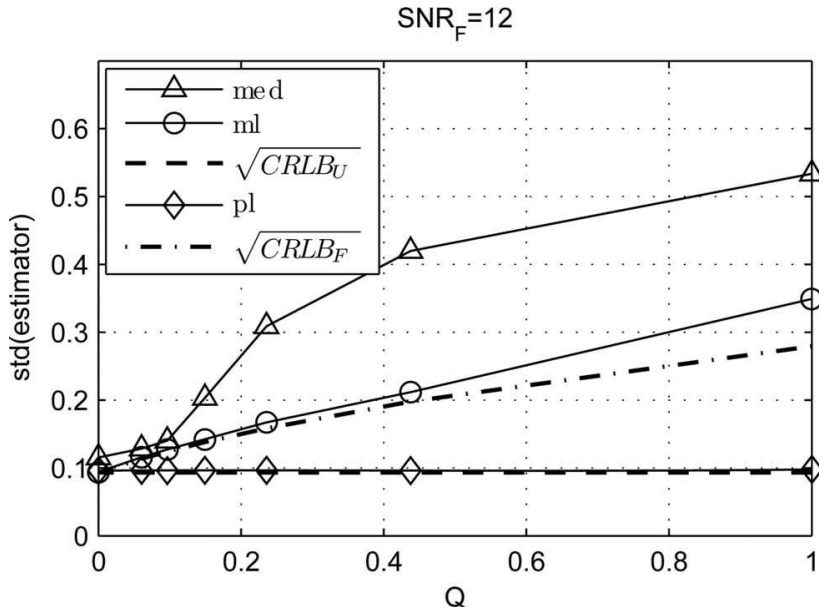

Fig. 5. Standard deviations $\sigma_{\text {median }}, \sigma_{m l}$, and $\sigma_{p l}$ of, respectively, the median, ML, and PL estimators for $S N R_{F}=12$ with the square roots of CRLB corresponding to the use of the uniform model $\left[C R L B_{U}\right.$; see Eq. (3)] and nonuniform model $\left[C R L B_{F}\right.$; see Eq. (16)] as a function of $Q$ [see Eq. (19)], which characterizes the nonuniformity of the illumination. The estimations were made by Monte Carlo simulations on $10^{4}$ realizations and nine samples with $P=0.5$. For $Q=0$, we estimate $\sigma_{m l}=0.114$ and $\sigma_{p l}=0.116$. 
Table 2. Values of $\boldsymbol{Q}^{a}$ and Gain in Precision Using the Nonuniform Model for Different Types of Illumination

\begin{tabular}{lcc}
\hline Type of Illumination & Value of $Q$ & Value of $s t d_{U}$ \\
\hline Uniform & 0 & $s t d_{F}$ \\
Uniformly & $\frac{1}{3(N-1)}$ & $(2 / \sqrt{3}) s t d_{F}$ \\
distributed & $\frac{1}{N_{-1}}$ & $\sqrt{2} s t d_{F}$ \\
Speckle & 1 & $\sqrt{N} s t d_{F}$ \\
Pulsed &
\end{tabular}

${ }^{a}$ See Eq. (19).

(2) Random uniformly distributed illumination: $\boldsymbol{F}$ is a random vector uniformly distributed between 0 and 1 . One knows that on average, its empirical mean is $m_{F}$ $=1 / 2$ and its empirical variance is $\sigma_{F}^{2} \approx 1 / 12$.

(3) "Speckle" illumination: $\boldsymbol{F}$ is distributed with an exponential PDF. In this case one knows that on average $\sigma_{F}^{2} / m_{F}^{2} \approx 1$.

(4) Pulsed illumination: $F_{j}=F^{0}$ and $F_{i}=0, \forall i \neq j$.

One obtains the values of $s t d_{U}$ listed in Table 2 . For $Q=0$ there is no potential improvement using the nonuniform model. Indeed, using the nonuniform model in this case implies the useless estimation of parameters $F_{i}$, which can decrease the actual precision. The improvement is modest for uniform distribution, more appreciable for exponentially distributed illumination, and does not depend on the number of samples. In the case of pulsed illumination, the improvement is significant and increases with the number of samples. We thus conclude that the nonuniform model will bring a modest precision improvement with "classical" nonuniform illumination distributions and a significant improvement with pulsed illumination.

\section{ACTUAL ESTIMATORS OF THE DOP IN THE PRESENCE OF NONUNIFORM ILLUMINATION}

We have studied in Section 4 the potential precision that can be reached under nonuniform illumination. We will now determine and characterize actual estimators of the DOP in this situation. We first consider the case when $\mathbf{F}$ is known, and then the case when it is unknown.

\section{A. Maximum-Likelihood Estimator with Nonuniform but Known Illumination \\ Our aim is here to determine the ML estimator of the DOP knowing the distribution of the illumination, that is, the vector $\boldsymbol{F}$. Starting with the expression of the log- likelihood in Eq. (13), we estimate $m_{x}$ and then $m_{y}$ by an- nulling its derivative with respect to $m_{x}$ and then $m_{y}$. One obtains $\hat{m}_{V}=\sum_{i=1}^{N} F_{i} V_{i} / \sum_{i=1}^{N} F_{i}$ with $V=X$ or $Y$. By the clas- sical property of invariance of the ML estimator with re- spect to parametrization, the expressions of $I$ and $P$ are, respectively,}

$$
\begin{gathered}
\hat{I}_{m l, F}=\frac{\sum_{i=1}^{N} F_{i}\left(X_{i}+Y_{i}\right)}{\sum_{i=1}^{N} F_{i}}, \\
\hat{P}_{m l, F}=\frac{\sum_{i=1}^{N}\left(F_{i} X_{i}-F_{i} Y_{i}\right)}{\sum_{i=1}^{N}\left(F_{i} X_{i}+F_{i} Y_{i}\right)} .
\end{gathered}
$$

It is easily shown that the estimator of $P$ can be written as

$$
\hat{P}_{m l, F}=\frac{S N R_{F} \times P+b_{1}^{\prime \prime}}{S N R_{F}+b_{2}^{\prime \prime}},
$$

where $b_{1}^{\prime \prime}$ and $b_{2}^{\prime \prime}$ are two Gaussian random variables with a mean of 0 and a variance of 1 . This expression is identical to that of the ML estimator in the case of uniform illumination: One has just replaced $S N R$ with $S N R_{F}$. As soon as the illumination is known, the PDF of the estimator and the CRLB thus have very similar expressions.

\section{B. Estimation When Illumination Is Unknown}

In most applications, $\mathbf{F}$ is a priori unknown. We must thus deal with a new estimation problem, where the data that have to be estimated are $\left\{\mathbf{F}, m_{X}, m_{Y}\right\}$. The estimation of $\mathbf{F}$ is not directly of interest, as our aim is to estimate the DOP: It is called a nuisance parameter. One can also note that $m_{X}$ and $m_{Y}$ cannot be estimated separately. Actually, the two vectors $\left\{\mathbf{F}, m_{X}, m_{Y}\right\}$ and $\left\{\mathbf{F} / a, m_{X} a, m_{Y} a\right\}$, where $a$ is any positive scalar, both correspond to a data sample $\chi^{\prime}$ [see Eq. (11)] with exactly the same statistical properties. This means that these two parameter sets cannot be distinguished from the observation of $\chi^{\prime}$. However, the DOP is invariant under multiplication of the data with a scalar $a$ and is thus the same for both aboveconsidered parameter vectors. It is thus likely that this parameter can be estimated from the data.

A way to deal with the nuisance parameters consists of considering them as deterministic unknowns and maximizing the likelihood with respect to those parameters: $\mathcal{L}_{p}\left(\chi^{\prime} \mid m_{X}, m_{Y}\right)=\arg \max _{\mathbf{F}}\left[\mathcal{L}\left(\chi^{\prime} \mid \mathbf{F}, m_{X}, m_{Y}\right)\right]$. The function obtained $\mathcal{L}_{p}$ is called profile likelihood (PL), since it is not really a likelihood function. Let us start with the expression of the log-likelihood $\ell$ of the $N$-sample $\chi^{\prime}$ in Eq. (13). We first estimate the $F_{i}$ by annulling the derivative of $\ell$ with respect to $F_{i}$ and obtain $\hat{F}_{i}=\left(X_{i} m_{X}+Y_{i} m_{Y}\right) /\left(m_{X}^{2}\right.$ $\left.+m_{Y}^{2}\right)$. Injecting these estimates into the expression of the log-likelihood, one obtains the profile log-likelihood

$$
\ell_{p}\left(\chi^{\prime} \mid P\right)=-2 N \ln (\sqrt{2 \pi} \sigma)-\frac{1}{2 \sigma^{2}} \sum_{1}^{N} \frac{\left[(P-1) X_{i}-(P+1) Y_{i}\right]^{2}}{1+P^{2}} .
$$

Annulling the derivative of $\ell_{p}$ with respect to $P$ leads to

$$
P^{2}-2 R P-1=0
$$

with $R=2\left(\Sigma_{1}^{N} X_{i} Y_{i}\right) /\left(\Sigma_{1}^{N} X_{i}^{2}-Y_{i}^{2}\right)$. 
Of the two solutions, we must keep the one that corresponds to a maximum of the likelihood. Studying the sign of $\partial^{2} \ell_{p} /(\partial P)^{2}$, which should be negative to obtain a maximum, one obtains

$$
\hat{P}_{p l}=-R+\operatorname{sign}\left[\sum_{i=1}^{N}\left(X_{i}^{2}-Y_{i}^{2}\right)\right] \sqrt{1+R^{2}} .
$$

This is the expression of the PL estimator of $P$. Before characterizing the performance of this estimator, it is interesting to determine on which signal parameters it depends.

From Eq. (24) it is seen that $\hat{P_{p l}}$ depends on $R$ and on $\operatorname{sign}\left[\sum_{i=1}^{N}\left(X_{i}^{2}-Y_{i}^{2}\right)\right]$. It is sufficient to analyze these values. After the cumbersome but easy computations detailed in Appendix $\mathrm{C}$, the random variable $R$ can be put into the form

$$
R=\frac{S N R_{F}^{2}\left(\frac{1-P^{2}}{2}\right)+S N R_{F}\left(b_{s}-P b_{d}\right)+\sqrt{N} b_{3}}{S N R_{F}^{2} P+S N R_{F}\left(b_{s}+P b_{d}\right)+\sqrt{N} b_{4}},
$$

where $b_{s}$ and $b_{d}$ are two Gaussian variables with zero mean and unit variance, and $b_{3}$ and $b_{4}$ are two random variables with zero mean and unit variance. One can deduce from this expression that $R$ depends only on $S N R_{F}$, $P$, and $N$, and so does the $\operatorname{sign}\left[\Sigma_{i=1}^{N}\left(X_{i}^{2}-Y_{i}^{2}\right)\right]$ (see Appendix C). The estimator $\hat{P}_{p l}$ therefore depends only on these three parameters and in particular not on $Q$, that is to say, on the illumination distribution. One can also anticipate that when the $S N R_{F}$ is sufficiently high, the precision of $\hat{P}_{p l}$ will not be very sensitive to $N$.

Let us illustrate these results with a Monte Carlo simulation performed on $10^{5}$ samples for a fixed $S N R_{F}$. We have represented in Fig. 6 the standard deviations of the estimators $\hat{P}_{m l, F}$ and $\hat{P}_{p l}$ as a function of $Q$. It is observed that they do not depend on $Q$ as predicted by Eqs. (21) and (25). The variations observed on the graph are simply due to fluctuations of estimation of standard deviation on a limited number of realizations shown by the error bars.

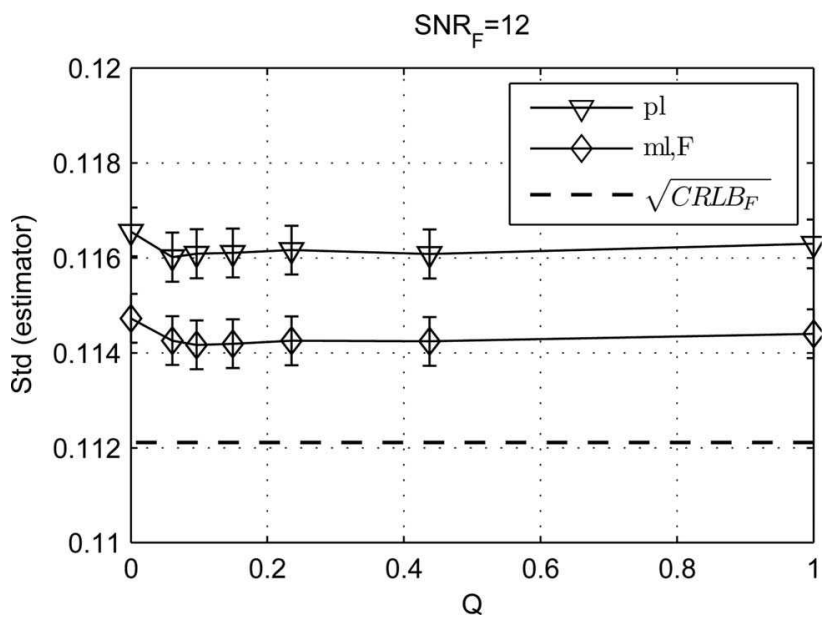

Fig. 6. Standard deviation of estimators $\hat{P}_{p l}$ and $\hat{P}_{m l, F}$ and the square root of the $C R L B_{F}$ [see Eq. (16)] as a function of $Q$ [see Eq. (19)], for $S N R_{F}=12, P=0.5$, and $N=9$. The estimations were made by Monte Carlo simulations on $10^{4}$ realizations.

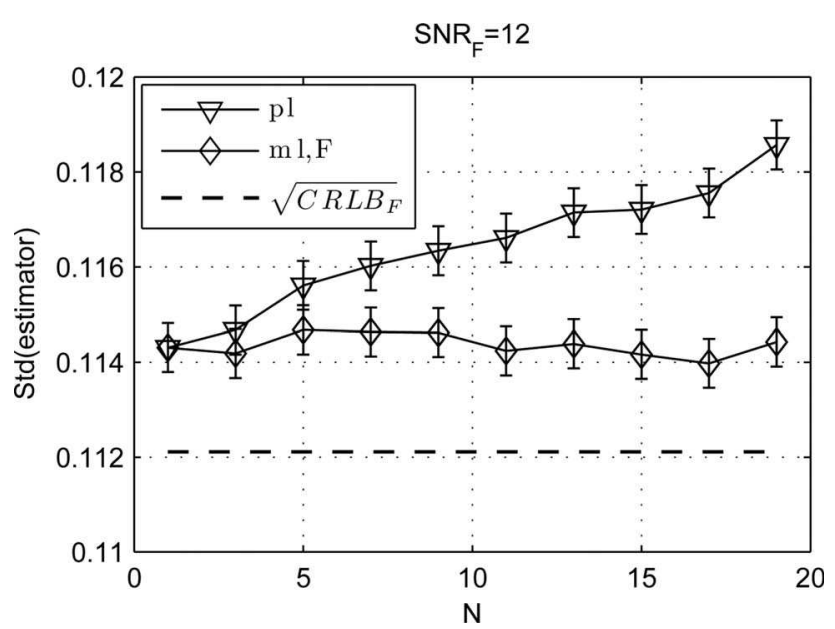

Fig. 7. Standard deviation of the estimators $\hat{P}_{p l}$ and $\hat{P}_{m l, F}$ and the square root of the $C R L B_{F}$ as a function of $N$ for $S N R_{F}=12$, $P=0.5$ and $Q=1$. The estimations were made by Monte Carlo simulations on $10^{5}$ realizations.

They correspond to $\pm 2 \sigma_{\text {std }}$, where $\sigma_{\text {std }}$ is the approximated standard deviation of the estimated standard deviation. In fact $\sigma_{\text {std }}$ is equal to the estimated standard deviation divided by $\sqrt{2 M}$, where $\mathrm{M}$ is the number of realizations used in the Monte Carlo simulation. We can also observe on Fig. 6 that the estimation performances are very close when the illumination is known (using $\hat{P}_{m l, F}$ ) and when it is unknown (using $\hat{P}_{p l}$ ), which means that not knowing the illumination distribution does not significantly degrade the estimation performance.

We have also characterized the dependency of $\hat{P}_{p l}$ on the number of samples in Fig. 7. As predicted, the CRLB and $\hat{P}_{m l, F}$ do not depend on the number of samples once $S N R_{F}$ is fixed (the slight fluctuations are once again due to the precision of the estimation of the values of interest with Monte Carlo simulations). It is observed that the standard deviation of $\hat{P}_{p l}$ slowly increases with the number of samples. This might seem surprising, but one must keep in mind that since $S N R_{F}$ is fixed, increasing $N$ means that the same SNR is spread over more pixels, and thus that more parameters $F_{i}$ must be estimated, which increases the estimator variance.

\section{Comparison with Estimators Adapted to Uniform Illumination}

It is interesting to compare $\hat{P}_{p l}$ with the estimators adapted to uniform illumination introduced in Section 2, that is, $\hat{P}_{\text {median }}, \hat{P}_{\text {em }}$, and $\hat{P}_{m l t}$. We define as in Section 3 a PL truncated estimator:

$$
\begin{aligned}
\hat{P}_{p l t} & =\hat{P}_{p l} \quad \text { if } \hat{P}_{p l} \in[0,1] ; \\
& =0 \text { if } \hat{P}_{p l}<0 \\
& =1 \text { if } \hat{P}_{p l}>1 .
\end{aligned}
$$

We have represented on Fig. 8 the empirical means and standard deviations of these estimators as a function of $S N R_{F}$. We have chosen $Q=1$, which corresponds to the 

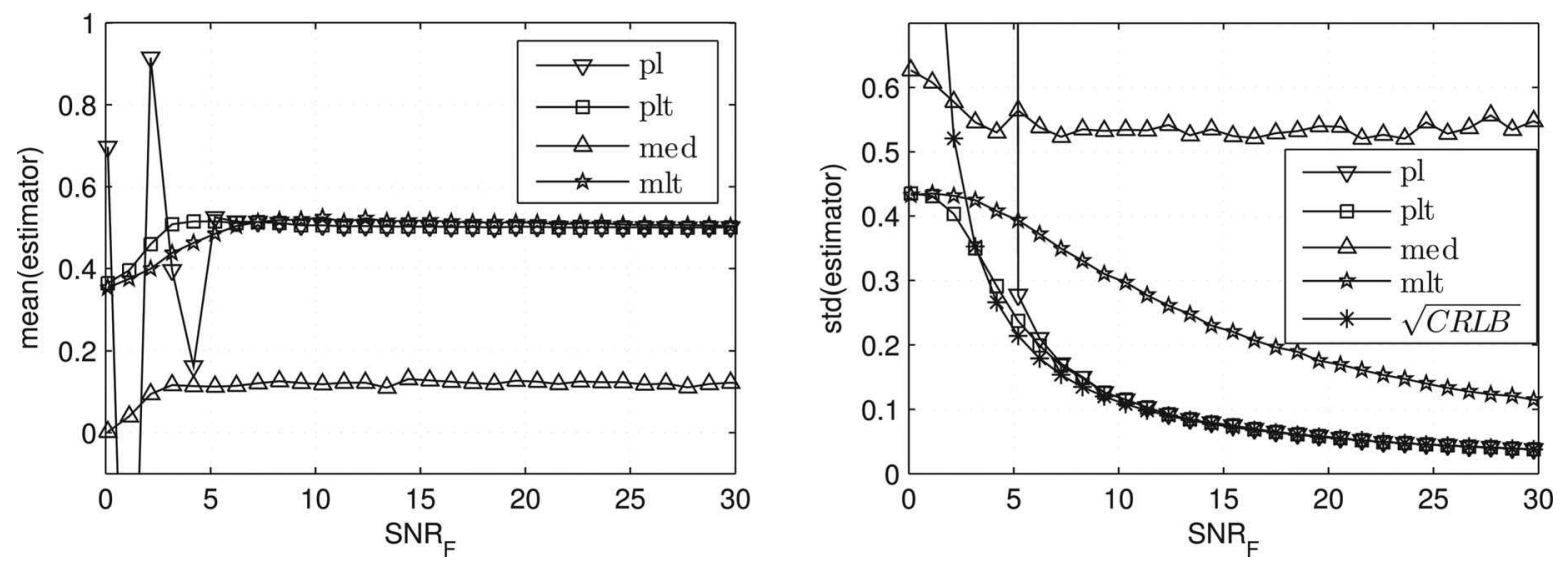

Fig. 8. Estimated means and standard deviations of estimators $\hat{P}_{p l}, \hat{P}_{p l t}, \hat{P}_{m e d}$, and $\hat{P}_{m l t}$ and the square root of CRLB as a function of $\mathrm{SNR}_{F}$ for $N=9, P=0.5$ and $Q=1$. The estimations are made with $10^{5}$ realizations.

most severe nonuniformity of illumination: All the SNR is concentrated in a single pixel of the sample. The median estimator is clearly not adapted to this value of $Q$. For SNR higher than 5 , the standard deviation of the PL estimator is lower than that of the truncated ML estimator and rapidly approches that of the $\mathrm{CRLB}$, where the $\mathrm{ML}$ estimator does not. It can be noted that $\hat{P}_{p l t}$ converges toward 0.36 just as $\hat{P}_{m l t}$ did. Regarding the truncation itself the same conclusion as in the uniform illumination case can be drawn: It improves the estimation performance for low values of the SNR and efficiently avoids aberrant results. It should thus be used as soon as the physical hypotheses are fulfilled.

We have represented in Fig. 5 the standard deviations $\sigma_{\text {median }}, \sigma_{m l}$, and $\sigma_{p l}$ of these estimators as a function of $Q$, which describes the uniformity of the illumination, for a given value of $S N R_{F}$. It is seen that the standard deviations of $\hat{P}_{\text {median }}$ and $\hat{P}_{m l}$ significantly depend on the illumination nonuniformity, whereas, as shown above, $\hat{P}_{p l}$ is absolutely insensitive to it. For low values of $Q, \hat{P_{m l}}$ is quite close to $\sqrt{C R L B_{U}}$. However, we notice that as $Q$ increases the standard deviation of $\hat{P}_{m l}$ becomes larger than $\sqrt{C R L B_{U}}$. Indeed, increasing $Q$ is just like decreasing the SNR corresponding to the uniform model, and we have seen in Section 3 that as the SNR decreases, the variance of actual estimators becomes larger than the CRLB.
For uniform illumination, which corresponds to $Q=0$, the PL estimator is only a little less precise than the ML estimator: Its standard deviation is estimated at $\sigma_{p l}$ $=0.116$ instead of $\sigma_{m l}=0.114$ for the ML estimator.

In conclusion, we have shown that the PL estimator is independent of illumination nonuniformity. Moreover, its performance is very close to that of the CRLB with known illumination. Consequently, it is a good alternative to standard ML estimation if one suspects that the illumination may be somehow spatially or temporally nonuniform.

\section{Experimental Results}

To illustrate these conclusions, we have performed the following experiments with the same setup as in Subsection 3.B. We consider the DOP images of pieces of homogenous transparent plastic on a white uniform diffusive background. The illumination is mainly focused on the first acquisition that corresponds to $Q \approx 1$. For that purpose we illuminate the scene during the first acquisition, and then switch it off for the remaining eight images. The results are represented on Fig. 9. The three objects are barely seen using the median and ML estimators. This subjective observation is confirmed by the standard deviation of the estimation of the DOP of the plastic, which decreases from 0.53 with $\hat{P}_{\text {med }}$ (a), to 0.13 with $\hat{P}_{m l t}$ (b), and 0.06 with $\hat{P}_{p l t}$ (c). Those results are in good agreement with the

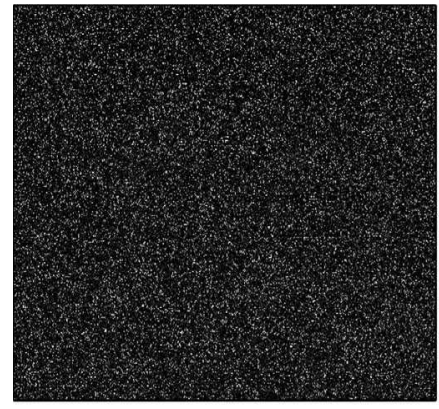

(a)

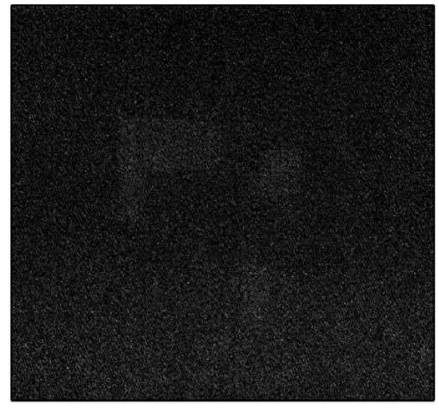

(b)

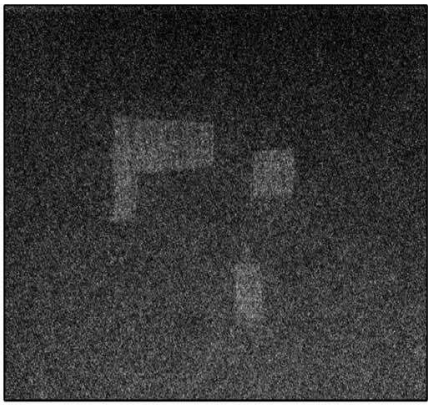

(c)

Fig. 9. Images of DOP estimated on nine successive images of the same scene with estimators $\hat{P}_{\text {med }}$ (a), $\hat{P}_{\text {mlt }}$ (b), and $\hat{P}_{\text {plt }}$ (c). The nonuniformity parameter $Q$ is close to 1 and $S N R_{F} \approx 23$. The scene is composed of pieces of transparent plastic on a diffusive white background obtained with a Basler A312f camera with a $70 \mathrm{~ms}$ exposure time. The DOP of the plastic and the background have been estimated, respectively, at 0.18 and 0.10 . 
simulation results in Fig. 8. With such a severe nonuniform illumination, the PL estimator indisputably shows the best performances.

\section{CONCLUSION}

We have addressed DOP estimation from intensity images perturbed by additive Gaussian noise when the materials present in the scene only depolarize the incident light. We have considered both uniform and nonuniform illuminations. We have demonstrated that with uniform illumination, truncating the estimators improves their precision and prevents aberrant results, as soon as the corresponding a priori hypotheses about the materials are fulfilled. Regarding the nonuniform illumination case, we have demonstrated that the estimation problem is similar to the uniform case when the spatial and temporal distribution of the illumination is known. If it is not, we have proposed a profile likelihood estimator that provides good performance. It is thus an interesting solution in cases where illumination fluctuates, for instance. An interesting perspective will consist in studying the detection performance for targets having a DOP contrast with the background.

\section{APPENDIX A: MEAN OF $\hat{\boldsymbol{P}}_{M L T}$ FOR SNR $=0$}

If $S N R=0$, one can write the pdf of $\hat{P}_{m l}$ as $\mathcal{P}_{m l}(\rho)=\mathcal{P}_{1}(\rho)$ $=1 / \pi\left(1+\rho^{2}\right)$. The probability that $\hat{P}_{m l}<0$ is $\int_{-\infty}^{0} \mathcal{P}(\rho)=1 / 2$. The probability that $\hat{P}_{m l}>1$ is $\int_{1}^{\infty} \mathcal{P}(\rho)=1 / 2-\tan ^{-1}(1) / \pi$. It is thus possible to write the pdf of $\hat{P}_{m l t}$ as

$$
\begin{aligned}
\mathcal{P}_{m l t}(\rho) & =\mathcal{P}_{m l}(\rho) \quad \text { if } \rho \in(0,1) \\
& =0 \text { if } \rho<0 ; \\
& =0 \text { if } \rho>1 ; \\
& =1 / 2 \text { if } \rho=0 ; \\
& =1 / 2-\tan ^{-1}(1) / \pi \text { if } \rho=1 .
\end{aligned}
$$

One then computes the mean of $\hat{P}_{m l t}$ and finds

$$
\left\langle\hat{P}_{m l t}\right\rangle=\int_{0}^{1} \rho \mathcal{P}_{m l t}(\rho) \mathrm{d} \rho=0+\int_{0}^{1} \rho \mathcal{P}_{m l}(\rho) \mathrm{d} \rho+\mathcal{P}_{m l t}(1) \approx 0.36 .
$$

The bias is thus equal to $b=\left\langle P_{m l t}\right\rangle-P=0.36-P$. This can be verified on Fig. 4.

\section{APPENDIX B: DEFINITION OF $C R L B_{U}$}

Let us assume that the sample $\chi=\{\boldsymbol{X}, \boldsymbol{Y}\}$ is observed under uniform illumination and thus follows the model in Eq. (2). The expression of the likelihood is

$$
\begin{aligned}
l(\chi \mid I, P)= & -2 N \ln (\sqrt{2 \pi} \sigma)-\frac{1}{2 \sigma^{2}} \sum_{1}^{N}\left[X_{i}-\frac{I(1+P)}{2}\right]^{2} \\
& -\frac{1}{2 \sigma^{2}} \sum_{1}^{N}\left[Y_{i}-\frac{I(1-P)}{2}\right]^{2}
\end{aligned}
$$

which can be written as $l(\chi \mid I, P)=f\left(S_{X}, S_{Y} \mid I, P\right)+g(\chi)$, where $g(\chi)$ is a function that does not depend on the parameters $I$ and $P$, and

$$
f\left(S_{X}, S_{Y} \mid I, P\right)=\frac{1}{2 \sigma^{2}}\left[S_{X} \frac{I(1+P)}{2}+S_{Y} \frac{I(1-P)}{2}\right]+\frac{I^{2}\left(1+P^{2}\right)}{2},
$$

with $S_{X}=\Sigma_{1}^{N} X_{i}$ and $S_{Y}=\Sigma_{1}^{N} Y_{i}$. In statistics language, $S_{X}$ and $S_{Y}$ are named sufficient statistics for the problem at hand, that is, once these two values are known, knowing the rest of the data $\chi$ is useless for purposes of estimation. In particular, the CRLB depends only on the statistical properties of $S_{X}$ and $S_{Y}$, which are Gaussian variables of means $N I(1+P) / 2$ and $N I(1-P) / 2$ and variances $N \sigma^{2}$.

Let us now assume that the actual data are nonuniformly illuminated and thus follow the model in Eq. (11), but the estimation is made by assuming uniform illumination. In this case, $S_{X}$ and $S_{Y}$ are Gaussian variables of means $F^{0} I(1+P) / 2$ and $F^{0} I(1-P) / 2$ and variances $N \sigma^{2}$. The situation is thus totally equivalent to having a uniformly illuminated sample with parameters $I^{\prime}=I F^{0} / N$ and $P$, that is, a SNR equal to [see Eq. (4) and Eq. (18)]

$$
S N R=\frac{I^{\prime} \sqrt{N}}{\sqrt{2} \sigma}=\frac{I F^{0}}{\sqrt{N} \sqrt{2} \sigma}=S N R_{F}^{\min } .
$$

Consequently, a lower bound on estimation variance using the uniform model in the presence of nonuniform illumination is given by the CRLB of the uniform model with SNR equal to $S N R_{F}^{\min }$ :

$$
C R L B_{U}=\frac{1+P^{2}}{\left[S N R_{F}^{m i n}\right]^{2}} .
$$

\section{APPENDIX C: PARAMETERS ON WHICH $R$ DEPENDS}

The expression of the PL estimator depends only on the quantity $R=2 \sum_{i=1}^{N} X_{i} Y_{i} / \Sigma_{i=1}^{N} X_{i}^{2}-Y_{i}^{2}$ and on $\operatorname{sign}\left[\Sigma_{i=1}^{N}\left(X_{i}^{2}\right.\right.$ $\left.-Y_{i}^{2}\right)$ ]. We will thus determine the parameters on which $R$ depends.

Let us analyze the numerator of $R$. Using the definition of the measured data in Eq. (11), one obtains after some computation

$$
\begin{aligned}
\sum_{i=1}^{N} X_{i} Y_{i}= & \left(\sum_{i=1}^{N} F_{i}^{2}\right) m_{x} m_{y}+\left(\sqrt{\sum_{i=1}^{N} F_{i}^{2}}\right) \sigma\left(m_{x} b_{y}+m_{y} b_{x}\right) \\
& +\sqrt{N} \sigma^{2} b_{3},
\end{aligned}
$$

where $b_{x}$ and $b_{y}$ are zero-mean Gaussian variables with unit variance, and $b_{3}$ is a zero-mean unit-variance random variable. We have used the fact that if $X$ and $Y$ are two independent random variables, then $\operatorname{var}(X Y)$ $=\operatorname{var}(X) \operatorname{var}(Y)$, where $\operatorname{var}($.$) denotes the variance.$ 
Let us now analyze the numerator. After some computation, one obtains

$$
\begin{aligned}
\sum_{i=1}^{N} X_{i}^{2}-Y_{i}^{2}= & \sum_{i=1}^{N} F_{i}^{2}\left(m_{x}^{2}-m_{y}^{2}\right)+2\left(\sqrt{\sum_{i=1}^{N} F_{i}^{2}}\right) \sigma\left(m_{x} b_{x}-m_{y} b_{y}\right) \\
& +2 \sqrt{N} \sigma^{2} b_{4},
\end{aligned}
$$

where $b_{4}$ is a zero-mean unit-variance random variable. We have used the fact that if $X$ is a Gaussian variable with zero mean and variance $\sigma^{2}$, then $\left\langle X^{2}\right\rangle=\sigma^{2}$ and $\operatorname{var}\left(X^{2}\right)=2 \sigma^{4}$.

Using the parametrization $(I, P)$ and the definition of $\mathrm{SNR}_{F}$ one has

$$
\begin{aligned}
& R=\frac{\left(S N R_{F}\right)^{2}\left(\frac{1-P^{2}}{2}\right)+S N R_{F}\left(b_{s}-P b_{d}\right)+\sqrt{N} b_{3}}{\left(S N R_{F}\right)^{2} P+S N R_{F}\left(b_{s}+P b_{d}\right)+\sqrt{N} b_{4}}, \\
& \operatorname{sign}\left(\sum_{i=1}^{N} X_{i}^{2}-Y_{i}^{2}\right)=\operatorname{sign}\left\{\sigma ^ { 2 } \left[\left(S N R_{F}\right)^{2} P\right.\right. \\
& \left.\left.+\operatorname{SNR}_{F}\left(b_{s}+P b_{d}\right)+\sqrt{N} b_{4}\right]\right\},
\end{aligned}
$$

where $b_{s}=\left(b_{x}+b_{y}\right) / \sqrt{2}$ and $b_{d}=\left(b_{x}-b_{d}\right) / \sqrt{2}$ are two Gaussian zero-mean and unit-variance variables.

It is seen that these two values depend only on parameters $S N R_{F}, P$, and $N$.

\section{ACKNOWLEDGMENTS}

Arnaud Bénière's Ph.D. thesis is supported by the Délégation Générale pour l'Armement (DGA), Mission pour la Recherche et l'Innovation Scientifique (MRIS), domain IMAT (contact: Jacques Blanc-Talon).

\section{References}

1. L. B. Wolff, "Polarization-based material classification from specular reflection," IEEE Trans. Pattern Anal. Mach. Intell. 12, 1059-1071 (1990).
2. S. L. Jacques, J. C. Ramella-Roman, and K. Lee, "Imaging skin pathology with polarized light," J. Biomed. Opt. 7, 329-340 (2002).

3. M. Alouini, F. Goudail, P. Réfrégier, A. Grisard, E. Lallier, and D. Dolfi, "Multispectral polarimetric imaging with coherent illumination: towards higher image contrast," Proc. SPIE 5432, 133-144 (2004).

4. S. Breugnot and P. Clémenceau, "Modeling and performances of a Polarization Active Imager at $\lambda$ $=806$ nm," Opt. Eng. (Bellingham) 39, 2681-2688 (2000).

5. F. Goudail and P. Réfrégier, "Statistical techniques for target detection in polarisation diversity images," Opt. Lett. 26, 644-646 (2001).

6. V. L. Gamiz and J. F. Belsher, "Performance limitations of a four-channel polarimeter in the presence of detection noise," Opt. Eng. 41, 973-980 (2002).

7. P. Réfrégier, F. Goudail, and N. Roux, "Estimation of the degree of polarization in active coherent imagery using the natural representation," J. Opt. Soc. Am. A 21, 2292-2300 (2004).

8. P. Réfrégier, M. Roche, and F. Goudail, "Cramer-Rao lower bound for the estimation of the degree of polarization in active coherent imagery at low photon level," Opt. Lett. 31, 3565-3567 (2006).

9. A. Bénière, F. Goudail, M. Alouini, and D. Dolfi, "Precision of degree of polarization estimation in the presence of additive Gaussian detector noise," Opt. Commun. 278, 264-269 (2007).

10. M. Alouini, F. Goudail, A. Grisard, J. Bourderionnet, D. Dolfi, I. Baarstad, T. Løke, P. Kaspersen, and X. Normandin, "Active polarimetric and multispectral laboratory demonstrator: contrast enhancement for target detection," Proc. SPIE 6396, 63960B (2006).

11. S. Breugnot and P. Clémenceau, "Modeling and performances of a polarization active imager at lambda =806 nm," Proc. SPIE 3707, 449-460 (1999).

12. P. Terrier and V. DeVlaminck, "Robust and accurate estimate of the orientation of partially polarized light from a camera sensor," Appl. Opt. 40, 5233-5239 (2001).

13. O. Germain and P. Réfrégier, "Snake-based method for the segmentation of objects in multichannel images degraded by speckle," Opt. Lett. 24, 814-816 (1999).

14. S. M. Kay, Fundamentals of Statistical Signal Processing Vol. I, Estimation Theory (Prentice-Hall, 1993).

15. D. V. Hinkley, "On the ratio of two correlated normal random variables," Biometrika 56, 635 (1969).

16. A. Papoulis, Probability, Random Variables and Stochastic Processes (McGraw-Hill, 1991). 